\title{
Trauma of the pancreas. A hidden disaster
}

\author{
Iulian Slavu*, Adrian Tulin*, Vlad Braga**, Octavian Enciu***, Dan Păduraru****, \\ Bogdan Socea*****, Lucian Alecu* \\ *"Prof. Dr. Agrippa lonescu" Clinical Emergency Hospital, Bucharest, Romania \\ **Clinical Emergency Hospital, Bucharest, Romania \\ ***"Elias" Clinical Emergency Hospital, Bucharest, Romania \\ $* * * *$ Bucharest University Emergency Hospital, Bucharest, Romania \\ *****"Sf. Pantelimon" Emergency Hospital, Bucharest, Romania
}

Correspondence to: Adrian Tulin, "Prof. Dr. Agrippa Ionescu" Clinical Emergency Hospital, Bucharest, 7 Ion Mincu Street, Bucharest, Romania, Mobile phone: +40752596675, E-mail: dr_2lin@yahoo.com

\begin{abstract}
Due to its anatomical position, traumatic lesions of the pancreas are rare and difficult to diagnose. Diagnosis time is paramount as an increase in duration translates into an increase in morbidity and mortality. Duct lesions are hard to identify on CT, ERCP being the investigation of choice but it is difficult to obtain as it requires highly specialized staff and equipment. Intraoperative macroscopic and palpatory evaluation of the pancreas in a trauma patient should be elective no matter what other lesions are present. The treatment is mainly dictated by this evaluation and is defined as it follows: stage I and II usually require a conservative approach; stage III, IV, and V usually imply resection of the pancreas although recent advances in conservative management have been made through ERCP and pancreatic duct stenting. In these stages, intraoperative evaluation should ensure the ampulla is intact if the head of the pancreas is not resected. Serum amylase and lipase levels do not offer a concrete direction towards a pancreatic lesion. Currently, there is no standard surgical treatment for these stages thus making intraoperative evaluation mandatory. One must remember that post-traumatic pancreatitis exists, which becomes apparent days after the accident.
\end{abstract}

Keywords: traumatology, pancreas, abdominal surgery

\section{Introduction}

Traumatic pancreatic lesions are rarely encountered and are usually associated with high-velocity impacts. Generally, at the first evaluation of trauma, the first abdominal organs inspected by the clinician are the spleen, kidney, and most frequently the liver, which also represents the largest abdominal organ. Through its retroperitoneal position and the multiple means of anchorage the pancreas is maintained in position but these do not prevent traumatic injury (Fig. 1) [1]. This retroperitoneal position is a two-edged sword because it protects the pancreas from the impact but makes it extremely difficult to diagnose, explore, and individualize the lesions (Fig. 1). Pancreatic damage is confirmed in only $2 \%$ of abdominal trauma [1].

It should also be noted that in $98 \%$ of the cases pancreatic trauma is associated with lesions of other abdominal organs and hence 
the difficulty of individualizing and diagnosing them (Fig. 1) [2].

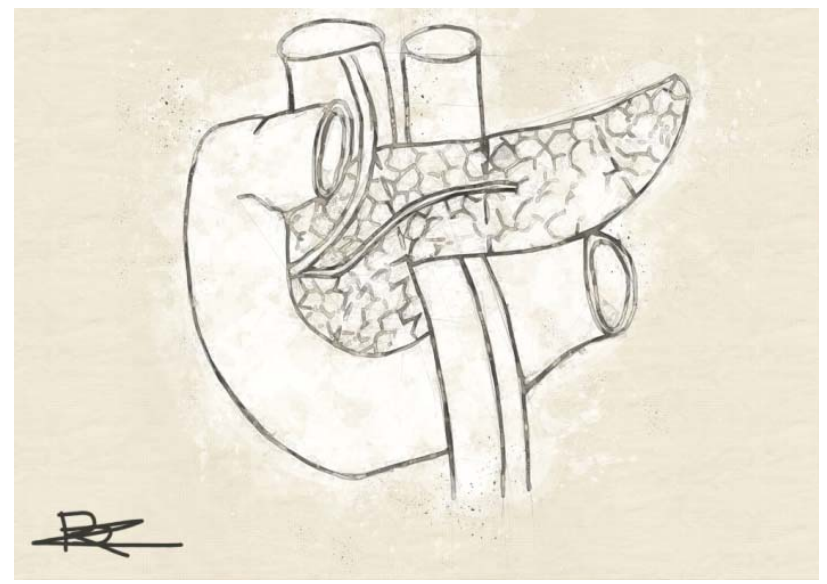

Fig. 1 Schematic description of the pancreas and the surrounding structures. This representation illustrates why a lesion at this level may have such dire complications for the patient

However, mortality in pancreatic trauma can reach $34 \%$, so this lesion should not be ignored and needs to be considered [3].

In the case of trauma involving the epigastric region, a high index of suspicion for pancreatic injury is required. Clinical signs such as Gray Turner or Cullen are useful when present but their absence does not rule out pancreatic injury [4].

It should also be noted that in the absence of acute complications, pancreatic trauma associates long-term complications such as fistulas, post-traumatic pancreatitis, or pancreatic abscess, respectively by contamination with intestinal flora [5].

For these patients, therapeutic decisions are not subject to strict guidelines and are tailored to the location of the lesion (head, neck, tail) and the status of the ductal system. In the case of restricted lesions and hemodynamically stable patients, endoscopic treatment may be attempted, but extended lesions require surgical intervention, exploration, and surgical resections.

Our study aims to draw attention to these lesions, the mechanism of production and treatment options by briefly reviewing the literature on this subject.

\section{Mechanism}

Any pancreatic lesion requires a high index of suspicion to be diagnosed. It is mandatory to be taken into account in the case of epigastric trauma caused by the impact of the car steering wheel, bicycle horns, or any blunt object that can transfer the kinetic energy to the epigastric region. The presence of neighboring anatomical structures of vital importance such as large vessels (Aorta, Inferior Vena Cava, portal vein, or superior mesenteric artery) increases the complexity of the lesions (Fig. 1). There are two types of mechanisms that the pancreas can be harmed by: penetrating mechanism, and contusion mechanism, in which an important amount of kinetic energy is transferred to this region. The distinction of the mechanism of injury is important as it guides the extent of exploration during surgery. Compression trauma usually associates lesions at the level of the immobile portion, namely the head of the pancreas, resulting in its destruction by explosion due to compression on the vertebral column that forms a posterior hard plane. Penetration injuries can occur at any level depending on the path of the cutting object $[5,6]$.

In terms of frequency distribution of these lesions, it appears that penetrating lesions are more common compared to those due to contusion [7].

\section{Classification of pancreatic lesions}

The comparison between different forms of treatment is difficult as isolated lesions are rare and the experience of different trauma clinics is limited. Although there are several staging schemes, a widely accepted form is the American Association for the Surgery of 
Trauma (AAST) scheme, which uses a graded system from I-V (Fig. 2).

Fortunately, the most severe injuries (V) are the rarest, accounting for less than $5 \%$ of the total $[8,9]$.

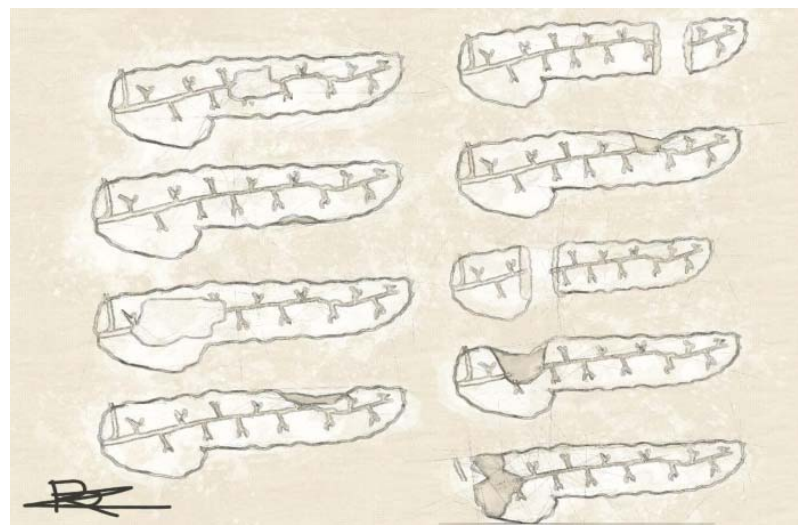

Fig. 2 The American Association for the Study of Trauma classification of pancreatic injuries presents five grades depending on the severity and location of parenchymal or duct disruption

- Grade 1-minor laceration/ contusion without duct injury

- Grade 2-major laceration or contusion without duct injury

- Grade 3-distal transection or duct injury

- Grade 4-proximal transection or parenchymal injury involving ampulla

- Grade 5-important transection or obliteration of pancreatic head

Another score that is still used but has not been able to prevail is the Organ Injury Score (OIS), which also captures the associated duodenal injury [10].

\section{Diagnostic}

\section{Abdominal X-Ray}

It is useful when associated lesions of neighboring organs exist, such as duodenal rupture that can be suggested by the presence of pneumoperitoneum. Usually, these gas bubbles are located at the level of the right psoas muscle, anterior to the kidneys, or overlapping the lumbar vertebrae. Air at any of these locations suggests a retroperitoneal organ injury [11].

\section{Abdominal ultrasound}

It has several advantages in the sense that it can be repeated, it is non-invasive, cheap, but it is operator dependent and it has serious limits for patients with large amounts of air in the colon or with important subcutaneous adipose tissue. It is useful as a first FAST assessment of the patient as it can identify free intraperitoneal fluid but it cannot identify the source or nature of the liquid (blood or intestinal content) [6].

\section{CT}

It is one of the basic investigations in the case of a trauma but a stable hemodynamic patient. It has the ability to identify $85 \%$ of the cases with a pancreatic injury. One aspect should be kept in mind, however, that the vast majority of pancreatic contusions or lesions are not CT - identifiable in the first hours after an accident. Also, it has limited utility in identifying pancreatic duct lesions [12].

\section{ERCP}

It represents a useful evaluation when pancreatic duct injury is uncertain on the CT investigation. The pancreatic duct injury should be considered because it is present in most cases for patients with a laceration of more than $50 \%$ of the diameter of the pancreas [13]. ERCP is an investigation that requires specialized equipment, a highly trained team, and quality radiological images. All these aspects transform this investigation in the case of a trauma patient, who is frequently hemodynamically unstable, hard to obtain. It can be performed in highly specialized centers even intraoperatively if a duct injury is suspected. 


\section{Laparotomy}

This option represents the last invasive method to diagnose a pancreatic injury. During a laparotomy in a trauma patient, one should thoroughly explore the pancreas as small lesions may go unnoticed. Keeping these aspects in mind, intraoperative ultrasound can be of real use. The pancreas must be carefully explored both on the anterior face, by entering the omental bursa and on the posterior face, by performing a Kocher maneuver, which should be extended towards the pancreas tail. If a pancreatic duct lesion is suspected, one may cannulate the Water Ampula after a duodenotomy and the main pancreatic duct and use a contrast substance and observe if extravasation follows [14]. In general, the vast majority of pancreatic lesions in a trauma patient are identified intraoperatively at exploration.

During initial exploration, one may identify certain signs of pancreatic lesions such as the presence of fluid in the omental bursa, biliary impregnation at the base of the transverse mesocolon, and the hepato-duodenal ligament associated with tissue crepitation. If any of these signs exist at laparotomy, a thorough exploration of the pancreas or the posterior face of the duodenum should be performed. If sufficient time has elapsed, cytosteatonecrosis spots produced by pancreatic enzymes may also be present. Attention should be paid to the depth of the laceration of the pancreas body - this being often indicative of a main duct injury if it is above $50 \%$ of the diameter of pancreas body [15].

Regarding the evaluation of blood biochemistry in a trauma patient with pancreatic injury, one must focus on serum amylase and lipase. The sensitivity of these enzymes is low and they increase in only $50 \%$ of the cases with pancreatic injury, while the absence of change in value represents a good indicator of the absence of a lesion [12]. It should be noted that both amylase and pancreatic lipase also increase in hemorrhagic shock.

\section{Treatment}

It is variable and largely dictated by the severity of the lesion. In the case of first and second-degree injuries, if there is a superficial hematoma or a parenchyma laceration that does not involve excretory ducts, minor contusions without alteration of the pancreatic duct, it is recommended to abstain from invasive maneuvers that can cause more harm than good $[\mathbf{1 6}, \mathbf{1 7}]$.

In all these situations, it is recommended to refrain from extensive resection or dissection, if there is no ductal involvement. One can use peripancreatic drainage and targeted bipolar hemostasis that should suffice. Hemostasis with the help of sutures or any other attempts to suture the pancreatic parenchyma can result in additional trauma due to the fact that when the knots are tightened, they transect the parenchyma or close a pancreatic duct by compression.

Treatment options become scarcer and more complicated in the case of grade III injuries. These lesions are frequently associated with important rupture or laceration, especially in the tail of the pancreas, which can go as far as to complete body transection. These lesions can also be treated by a conservative approach. One may use peripancreatic drainage with negative pressure or, in advanced trauma, if the situation requires, a spleno-pancreatectomy can be performed [18].

Splenectomy is not always mandatory and the spleen should be preserved as much as possible in a young patient or child due to the immune function it provides. The decision to preserve the spleen is difficult due to the fact that the pancreaticosplenic ligament must be dissected to have access to the pancreas, which involves a considerable length of time, 
time that is not always available in a trauma patient [18]. If the patient is hemodynamically unstable, distal spleno-pancreatectomy should be performed. The remaining pancreatic stump can be sutured with separate threads or resected and closed with the help of a stapler. There is no concrete data to point to any of the techniques. If it is possible, one should strive to individually close each pancreatic duct [19].

Grade IV and V lesions usually associate duodenal laceration. In these situations, the surgical treatment is more complex but again the local evaluation is of primary importance and dictates the therapeutic gestures. Thus, in the case of advanced lesions of the duodenum with important devascularization, respectively with massive tissue destruction of the pancreas head, duodenopancreatectomy will be required.

A complex intervention in a traumatized patient is not preferable but in the absence of other options it needs to be performed. If the pancreas shows mild tissue damage with minimal ductal involvement, one can opt for minimal conservative treatment, which will consist of hemostasis and local necrosectomy. Later in the postoperative period, ERCP can be performed with stenting of the pancreatic duct. To be able to implement this treatment, the operator must ensure the vascular viability of the ampulla. This is best done through a duodenotomy. If the duodenum is viable and there is a complete transection of the pancreas, another option is to close the proximal pancreatic stump (with separate threads or stapler) and the distal stump will be anastomosed to a segment of the digestive tract: stomach or small intestine. This procedure was developed due to the fact that patients often developed diabetes after total pancreatectomy.

In conclusion, traumatic pancreatic injuries are rare but imply an important morbidity. They are difficult to diagnose and a high index of suspicion needs to exist. CT is the mainstay investigation to identify the pancreas.
Treatment solutions are multiple and range from a conservative approach, which consists of local drainage and duct stenting through ERCP, to spleno-pancreatectomy or duodenopancreatectomy. It is imperative to observe the state of the ductal distal.

\section{Conflict of Interest statements}

Authors state no conflict of interest.

\section{Informed Consent and Human and Animal Rights statements \\ Informed consent has been obtained from all individuals included in this study.}

\section{Authorization for the use of human subjects}

Ethical approval: The research related to human use complies with all the relevant national regulations, institutional policies, is in accordance with the tenets of the Helsinki Declaration, and has been approved by the authors' institutional review board or equivalent commitee.

\section{References}

1. Cirillo RL, Koniaris LG. Detecting blunt pancreatic injuries. J Gastrointest Surg. 2002; 6:587-598, PMID: 12127126.

2. Jurkovich GJ, Carrico CJ. Pancreatic trauma. Surg Clin North Am. 1990; 70:575-93.

3. Schurink GW, Bode PJ, van Luijt PA, van Vugt $A B$. The value of physical examination in the diagnosis of patients with blunt abdominal trauma: a retrowspective study. Injury. 1997; 28:261-265, PMID: 9282178.

4. Asensio JA, Forno W. Duodenal Injuries. In: Demetriades D, Asensio JA, eds. Trauma Management. 2000, Austin, TX, Landes Bioscience, 354-360.

5. Degiannis E, Glapa M, Loukogeorgakis SP, Smith MD. Management of pancreatic trauma. Injury. 2008; 39(1):21-29.

6. Fisher M, Brasel K. Evolving management of pancreatic injury. Curr Opin Crit Care. 2011; 17:613-7.

7. Seamon MJ, Kim PK, Stawicki SP, Dabrowski GP, Goldberg AJ, Reilly PM et al. Pancreatic injury in damage controllaparotomies: is pancreatic resection safe during the initial laparotomy? Injury. 2009; 40:61-5.

8. lacono C, Zicari M, Conci S, Valdegamberi A, de Angelis $M$, Pedrazzani $C$ et al. Management of pancreatic trauma: a pancreatic surgeon's point of view. Pancreatology. 2016; 16:302-8. 
9. Moree EE, Cogbill TH, Malangoni MA, Jurkovich GJ, Champion HR, Gennarelli TA et al. Organ injury scaling, II: pancreas, duodenum, small bowel, colon and rectum. J Trauma. 1990; 30:1427-9.

10. Jurkovich GJ, Carrico CJ. Pancreatic trauma. Surg Clin N Am. 1990; 70:575-593.

11. Cirillo RL Jr, Koniaris LG. Detecting blunt pancreatic injuries. J Gastrointest Surg. 2002; 6:587-598.

12. Vijay A, Abdelrahman H, El-Menyar A, Al-Thani H. Early laparoscopic approach to pancreatic injury following blunt abdominal trauma. J Surg Case Rep. 2014; 12:1-3.

13. Subramanian A, Dente CJ, Feliciano DV. The management of pancreatic trauma in the modern era. Surg Clin N Am. 2007; 87:1515-32.

14. Gomez D, Addison A, De Rosa A, Brooks A, Cameron IC. Retrospective study of patients with acute pancreatitis: is serum amylase still required? BMJ Open.2012;2:e001471

15. Takishima T, Sugimoto K, Hirata M, Asari Y, Ohwada T, Kakita A. Serum amylase level on admission in the diagnosis of blunt injury to the pancreas: its significance and limitations. Ann Surg. 1997; 226(1):70-6.

16. Cerwenka $H$, Bacher $H$, El-Shabrawi $A$, Kornprat $P_{\text {, }}$ Lemmerer $M$, Portugaller $\mathrm{HR}$ et al. Management of pancreatic trauma and its consequences - guidelines or individual therapy? Hepatogastroenterology. 2007; 54:581-4.

17. Fabian T, Kudsk K, Croce M, Payne L, Mangiante E, Voeller $G$ et al. Superiority of closed suction drainage for pancreatic trauma: A randomized prospective study. Ann Surg. 1990; 211(6):724-8.

18. Knaebel HP, Diener MK, Wente MN, Buchler MW, Seiler $\mathrm{CM}$. Systematic review and meta-analysis of technique for closure of the pancreatic remnant after distal pancreatectomy. Br J Surg. 2005; 92(5):539-46.

19. Stringer MD. Pancreatitis and pancreatic trauma. Semin Pediatr Surg. 2005; 14:239-246. 